\title{
COMMENTARY
}

\section{Flagellar oscillation: new vibes from beads}

\author{
CHARLES J. BROKAW \\ Division of Biology, Califomia Institute of Technology, Pasadena CA 91125, USA
}

\section{Introduction: a mechanochemical oscillator}

The oscillatory mechanism of eukaryotic flagella and cilia remains one of the mysteries of cell biology. It has been difficult to dissect the oscillation functionally when its coupling to the bending of the flagellum has been required to provide a read-out of the functioning of the oscillator. A way around this difficulty is now provided by work from several laboratories, involving the attachment of submicrometer diameter beads to microtubules as indicators of dynein-driven movement of microtubules.

No one considers the flagellar oscillator to be a purely biochemical oscillator, since its frequency, and other properties of flagellar bending waves, can be significantly altered by external mechanical influences (reviewed by Brokaw, 1989a). Flagella can be described as highpowered mechanochemical oscillators that operate at the subcellular level. The oscillatory mechanism of the dynein-microtubule system in flagella is therefore comparable to that of the myosin-actin system of insect fibrillar flight muscle (Pringle, 1957). However, flagella oscillate at frequencies ranging from less than 1 cycle per second to more than 100 cycles per second, with microtubule sliding amplitudes of $100 \mathrm{~nm}$ or more, while fibrillar muscles oscillate at frequencies up to 1000 cycles per second, but with local amplitudes usually of the order of $10 \mathrm{~nm}$.

Following the lead of Machin (1958), the idea of a mechanochemical oscillator in flagella evolved into the proposal that the active sliding process is controlled by the curvature of the flagellum (Brokaw, 1971). Computer simulations have shown that this control specification can generate oscillation and bending wave propagation in flagellar models, but is insufficient to determine completely the characteristics of the resulting movement (Brokaw, 1972, 1985).

Alternatively, the mechanochemical oscillator might be a mechanism for producing oscillatory sliding of microtubules, independently of flagellar bending. The flagellum could then be described as a system of distributed shear oscillators, strongly coupled by the continuity of the microtubules. Models of dynein cross-bridge behavior that could generate oscillatory sliding have been proposed (Brokaw, 1975; Murase and Shimizu, 1986).

One might expect that if the flagellar oscillator were an oscillatory sliding mechanism, oscillatory sliding should occur in fragments of axonemes that do not display bending, such as fragments produced by shearing suspensions of demembranated sea urchin sperm flagella. Indirect evidence against such oscillatory sliding was obtained by measurements of ATP dephosphorylation by suspensions of broken, demembranated sea urchin spermatozoa. Breakage greatly reduced the rate of ATP dephosphorylation, to a level close to that of trypsindisintegrated axonemes (Brokaw and Benedict, 1968; Gibbons and Gibbons, 1972; Brokaw and Simonick, 1977). A more direct approach is now possible using submicrometer diameter beads attached to microtubules as indicators of microtubule sliding. Initially, this technique was used to confirm, by direct observations of the sliding of outer doublet microtubules in ATP-reactivated, demembranated sea urchin sperm flagella, that flagellar bending is associated with sliding of outer doublet microtubules (Brokaw, 1988, 1989b). It was recognized that this technique could also be used with fragments of axonemes, to look for oscillatory sliding (Brokaw, 1989b, and unpublished observations; Kamimura and Kamiya, 1989). These observations directly demonstrated the absence of oscillatory sliding with amplitudes of more than $50 \mathrm{~nm}$ that would correspond to the oscillatory sliding that occurs in a beating flagellum. One might reasonably conclude that bending of the flagellum is an essential component of flagellar oscillation.

\section{Hyperosclllations}

However, nature is not so simple. The highly sensitive measurement techniques used by Kamimura and Kamiya (1989) allowed them to discover a low amplitude $(\sim 4 \mathrm{~nm}$ ), high frequency (up to $340 \mathrm{~Hz}$ ) oscillation of beads attached to the outer doublet microtubules of fragments of demembranated sea urchin sperm flagella. The frequency of this oscillation varied with MgATP concentration in the same manner as the beating frequency of demembranated sea urchin sperm flagella. This ATP-dependence rules out artifacts and suggests that the bead movements are reliable indicators of an active, oscillatory sliding of the microtubules to which they are attached.

When $0.5 \mu \mathrm{M}$ vanadate (a potent inhibitor of dyneindriven motility) was added, this oscillatory movement 
disappeared, and was replaced by an aperiodic, back-andforth diffusion of the bead, with a similar range of displacements. This situation appears to be an example of a classic physical situation, often described in elementary physics texts as 'Brownian motion of a galvanometer' (e.g. see Sears, 1953). This is a system with one degree of freedom that undergoes a random walk, or diffusion, in the presence of an elastic force that constrains it to remain near the neutral position of the elastic restraint. For simple linear restraints, the restraining force constant, $k_{\mathrm{F}}$, can be calculated from:

$$
<x^{2}>=k_{\mathrm{B}} T / k_{\mathrm{F}}
$$

where $\left\langle x^{2}\right\rangle$ is the mean square displacement measured for long time intervals, $k_{\mathrm{B}}$ is Boltzmann's constant, and $T$ is absolute temperature. Although no quantitative calculations of $\left\langle x^{2}\right\rangle$ are presented, Fig. 1b of Kamimura and Kamiya (1989) suggests that an order of magnitude estimate of $\left\langle x^{2}\right\rangle$ might be about $(2 \mathrm{~nm})^{2}$, leading to an estimate of about $1 \mathrm{pN} \mathrm{nm}^{-1}$ for $k_{\mathrm{F}}$. This value is a measure of the elastic resistance to sliding of microtubules within the axoneme, previously referred to as elastic shear resistance, $E_{\mathrm{S}}$ (Brokaw, 1972). This elastic shear resistance is considered to result from elastic linkages between the outer doublet microtubules, independent of the dynein cross-bridges. $E_{\mathrm{S}}$ is a factor relating bending moment, $M$, and shear angle, $\sigma$ :

$$
\mathrm{d} M / \mathrm{d} s=E_{\mathrm{S}} \sigma,
$$

with a value for $E_{\mathrm{S}}$ of $6 \mathrm{pN}$ as estimated previously (Omoto and Brokaw, 1982). If the bottom of an axoneme is attached to the slide surface and a bead is riding on a doublet microtubule at the top of the axoneme, about $180 \mathrm{~nm}$ from the surface, the conversion between $k_{\mathrm{F}}$ and $E_{\mathrm{S}}$ is:

$$
k_{\mathrm{F}} * 180=E_{\mathrm{S}} * \text { length of axoneme/180. }
$$

This leads to an estimate of $E_{\mathrm{S}}=3 \mathrm{pN}$ if the length of the axoneme is $10 \mu \mathrm{m}$, in reasonable agreement with previous estimates. Further study of the bead movements seen by Kamimura \& Kamiya under these conditions may lead to a more refined estimate for $E_{\mathrm{S}}$. Data on $k_{\mathrm{F}}$ for axonemes of various lengths will test the assumption that there are no changes in lengths of outer doublet microtubules during these movements.

\section{Mlcrotubules unleashed}

In a totally different situation, Vale et al. (1989) have described the diffusion of microtubules over a surface coated with the flagellar ATPase, dynein, in the presence of vanadate and either ATP or ADP. The movement was quantitated by image analysis of video images of gold beads attached to the microtubules. Using a dynein fragment containing the $\beta$ heavy chain and intermediate chain 1 ( $\beta$-IC1), the diffusion coefficient for longitudinal movement was about $10 \%$ of that expected for Brownian movement, calculated by assuming that the resistance to movement was just the hydrodynamic resistance. With intact outer arm dynein, the diffusion coefficient was lower still, by a factor of about 6 . In the presence of ATP, without vanadate, the movement becomes a smooth, unidirectional gliding of the microtubules, with slightly higher velocities for $\beta$-IC1 than for intact outer arm dynein (Sale and Fox, 1988).

The most striking feature of this movement was its restriction to the direction parallel to the microtubule. Diffusion perpendicular to the microtubule occurred with a diffusion coefficient that was at least two orders of magnitude less. This difference suggests that the dyneinADP-vanadate complexes that are bound loosely to the microtubule and retain it close to the surface are able to slide freely along the microtubule, without detaching and reattaching, or shifting laterally on the microtubule (Vale et al. 1989). This information is important because the dynein-ADP-vanadate complex is believed to be an analog of the dynein-ADP-phosphate complex that occurs normally in the dynein ATPase cycle. The release of inorganic phosphate from this complex is believed to occur in association with transformation of the weak dynein-microtubule interaction to a strong, force-producing cross-bridge.

Since the diffusion coefficient measured for this movement by Vale et al. (1989) is less than the value calculated for unrestricted diffusion of a microtubule, it provides information about the sliding resistance resulting from the weak attachment of dyneins to the microtubule. Modulation of this sliding resistance might be a significant component of the oscillatory mechanism. At least two caveats should be mentioned. Vale et al. obtained the weakly attached state only at low ionic strengths (less than $0.1 \mathrm{M} \mathrm{KCl}$ ) that are well below the optimum for reactivated movement of demembranated sea urchin sperm flagella. In the experiments of Vale et al., dynein is interacting with the surface lattice of microtubules polymerized from brain tubulin, rather than the surface lattice of the specialized B-tubules with which it interacts within the axoneme. Nevertheless, a method now exists for learning more about the properties of this state in this simplified, in vitro, system.

\section{Back to the axoneme}

The bead movements detected by Kamimura and Kamiya (1989) may also provide information about the weakly bound dynein cross-bridge state, since analysis of the diffusion of an attached bead for very short time intervals provides information about the frictional resistance to microtubule sliding, just as in the experiments of Vale et al. (1989). In the absence of vanadate, the movement of the attached bead changes to the regular, high-frequency oscillation described by Kamimura and Kamiya (1989), just as the Brownian movement of a galvanometer changes to oscillation at its natural frequency when the frictional damping is reduced (Sears, 1953). Vanadate could inhibit movement by increasing the frictional resistance, by increasing the fraction of dyneins trapped in the weakly bound cross-bridge state.

It has been tempting to start thinking about flagellar oscillation in the same way that one thinks about the 
oscillation of familiar physical systems, such as a mass attached to a spring. However, the size scale of flagella is such that the inertial effects of mass are negligible compared to viscous resistances of the medium, so that mass cannot be a component of the flagellar oscillatory mechanism. For example, the natural frequency, $f$, of a simple harmonic oscillator is given by:

$$
2 \pi f=\left(k_{\mathrm{F}} / m\right)^{i}
$$

where $m$ represents its mass. For a frequency of $300 \mathrm{~s}^{-1}$ and $k_{\mathrm{F}}=1 \mathrm{pN} \mathrm{nm}{ }^{-1}$, the mass has to be $0.25 \times 10^{-9} \mathrm{~kg}$, or about the mass of a cube of water that measures $63 \mu \mathrm{m}$ on a side. There is no way that this is possible for a submicrometer diameter bead attached to a fragment of an axoneme!

The conclusion that mass cannot be a component of the flagellar oscillator leads to the idea that the flagellum contains some analog of momentum that keeps the active driving force turned on as the system passes through the position of zero elastic force, and does not allow the system to come to rest at equilibrium. This, in fact, is what is achieved by the curvature-control mechanism (Brokaw, 1971) or the shear oscillation mechanisms of Brokaw (1975) and Murase and Shimizu (1986).

If the elastic shear resistance calculated previously is a part of the mechanism of the high-frequency low-amplitude oscillation seen by Kamimura \& Kamiya, the forces involved in driving this oscillation would be in the range of 1 to $10 \mathrm{pN}$, which is just the range estimated for the force produced by a single dynein cross-bridge! (reviewed by Brokaw and Johnson, 1989.) It is difficult to imagine a mechanism that could produce such regular oscillations from the probabilistic behaviour of single dynein molecules. If, on the other hand, regularity of the oscillation results from the cooperative activity of many dyneins, the forces resulting from elastic shear resistance will be irrelevant. All of the oscillatory mechanism must then reside in the dynein-microtubule interactions, leading to the conclusion that flagella contain an oscillator that is not only massless but also springless. Fortunately, these alternatives can be tested by examining the effects of digestion with elastase, which allows larger amplitudes of flagellar bending (Brokaw, 1980), but should have no effect on a low-amplitude oscillation that does not involve the elastic shear resistance.

The observations of Kamimura \& Kamiya also suggest that it may be too soon to abandon the idea that the flagellar oscillator is a shear oscillator. A flagellum that is a collection of distributed shear oscillators must contain a mechanism to ensure that these oscillators function metachronously, to produce propagated bends, rather than synchronously. Synchronous oscillation would simply wave the basal end of the flagellum back and forth, without producing useful motion. The absence of largeamplitude shear oscillations in fragments of flagella may simply indicate that the mechanisms that have evolved to prevent synchronous shear oscillation are strong enough to survive breakage of the flagellum. The challenge now is to try and understand what the high-frequency lowamplitude oscillatory capability and the ninefold construction of the axoneme are telling us about how the flagellum ensures metachronous, rather than synchronous, shear oscillation.

\section{Conclusion: the method is the message}

This new work has combined a simple idea - the attachment of submicrometer diameter beads to microtubules to provide indicators of microtubule position with a variety of new technologies. In my laboratory, bead labelling has been used in conjunction with computerized image analysis of high-speed photomicrographs (Brokaw, 1989b, 1990). Vale et al. (1989) have used it in conjunction with video microscopy and computerized image analysis of video records (see also Gelles et al. 1988). Kamimura and Kamiya (1989) have used a highprecision analog detector for microscopic movements of a bead (Kamimura, 1987). In each case, we have seen only the first results from new techniques that will continue to be major sources of information about flagellar oscillation and other forms of biological motility.

My work has been generously supported by a grant from the National Institutes of Health, no. GM 18711.

\section{References}

Brokaw, C. J. (1971). Bend propagation by a sliding filament model for flagella. 7. exp. Biol. 55, 289-304

BrokAw, C. J. (1972). Computer simulation of flagellar movement. I. Demonstration of stable bend propagation and bend initiation by the sliding filament model. Biophys. J. 12, 564-586.

BroKaw, C. J. (1975). Molecular mechanism for oscillation in flagella and muscle. Proc. natn. Acad. Sci. U.S.A. 72, 3102-3106. Brokaw, C. J. (1980). Elastase digestion of demembranated sperm flagella. Science 207, 1365-1367.

Brokaw, C. J. (1985). Computer simulation of flagellar movement. VI. Simple curvature-controlled models are incompletely specified. Biophys. 7. 48, 633-642.

Brokaw, C. J. (1988). Direct measurement of sliding between outer doublet microtubules in actively beating sperm flagella (Abstract) Cell Motil. Cytoskel. 11, 212-213.

Brokaw, C. J. (1989a). Operation and regulation of the flagellar oscillator. In Cell Movement, vol. 1, The Dynein ATPases (ed. F. D Warner, P. Satir and I. R. Gibbons), pp. 267-279. New York: A. R. Liss.

Brokaw, C. J. (1989b). Direct measurements of sliding between outer doublet microtubules in swimming sperm flagella. Science $243,1593-1596$

Brokaw, C. J. (1990). Descriptive and mechanistic models of flagellar motility. In Biological Motion (ed. W. Alt and G. Hoffmann). Berlın: Sprınger-Verlag (ın press).

BROKAW, C. J. AND BENEDICT, B. (1968). Mechanochemical coupling in flagella. I. Movement-dependent dephosphorylation of ATP by glycerinated flagella. Archs Biochem. Biophys. 125, 770-778.

Brokaw, C. J. AND Johnson, K. A. (1989). Dynein-induced microtubule sliding and force generation. In Cell Movement, vol. 1, The Dynein ATPases (ed. F. D. Warner, P. Satir and 1. R. Gibbons), pp. 191-198. New York: A. R. Liss.

Brokaw, C. J. AND Simonick, T. F. (1977). Nechanochemical coupling in flagella. V. Effects of viscosity on movement and ATP. dephosphorylation of Triton-demembranated sea urchin spermatozoa. I. Cell Sci. 23, 227-241.

Gelles, J., SchnapP, B. J. ANd Scheetz, M. P. (1988). Tracking kinesin-druven movement with nanometer-scale precision. Nature, Lond. 331, 450-453.

Gibbons, B. H. And Gibbons, I. R. (1972). Flagellar movement and adenosine triphosphatase activity in sea urchin sperm extracted with Triton X-100. \%. Cell Biol. 54, 970-985.

Kamimura, S. (1987). Direct measurement of nanometric 
displacement under an optical microscope. Appl. Opt. 26 , 3425-3427.

KamimuRA, S. ANd KamiYA, R. (1989). High-frequency nanometrescale vibration in 'quiescent' flagellar axonemes. Nature, Lond. 340, 476-478.

MACHin, K. E. (1958). Wave propagation along flagella. J. exp. Biol. $35,796-806$

Murase, M. and Shimizu, H. (1986). A model of flagellar movement based on cooperative dynamics of dynein-tubulin crossbridges. I. theor. Biol. 119, 409-433.

Oмото, C. K. and Brokaw, C. J. (1982). Structure and behaviour of the sperm terminal filament. 7 . Cell Sci. 58, 385-409.
Pringle, J. W. S. (1957). Myogenic rhythms. In Recent Advances in Invertebrate Physiology (ed. B. T. Scheer), pp. 99-115. Eugene, OR: Unuversity of Oregon Publications.

Sale, W. S. And Fox, L. A. (1988). The isolated $\beta$-heavy chain subunit of dynein translocates microtubules in vitro. 7. Cell Biol. 107, 1793-1798.

SEARS, F. W. (1953). An Introduction to Themodynamics, The Kinetic Theory of Gases, and Statistical Mechanics. p. 352. Cambridge, MA: Addison-Wesley Publ. Co.

Vale, R. D., Soll, D. R. and Gibbons, I. R. (1989). Onedimensional diffusion of microtubules bound to flagellar dynein. Cell 59, 915-925. 